\title{
Variational Principle underlying Scale Invariant Social Systems
}

\author{
A. Hernando ${ }^{1}$, A. Plastino ${ }^{2,3}$ \\ 1 Laboratoire Collisions, Agrégats, Réactivité, IRSAMC, \\ Université Paul Sabatier 118 Route de Narbonne 31062 - Toulouse CEDEX 09, France \\ ${ }^{2}$ National University La Plata, IFLP-CCT-CONICET, C.C. 727, 1900 La Plata, Argentina \\ ${ }^{3}$ Universitat de les Illes Balears and IFISC-CSIC, 07122 Palma de Mallorca, Spain
}

\begin{abstract}
MaxEnt's variational principle, in conjunction with Shannon's logarithmic information measure, yields only exponential functional forms in straightforward fashion. In this communication we show how to overcome this limitation via the incorporation, into the variational process, of suitable dynamical information. As a consequence, we are able to formulate a somewhat generalized Shannonian Maximum Entropy approach which provides a unifying "thermodynamic-like" explanation for the scale-invariant phenomena observed in social contexts, as city-population distributions. We confirm the MaxEnt predictions by means of numerical experiments with random walkers, and compare them with some empirical data.
\end{abstract}

\section{INTRODUCTION}

Scale-invariant phenomena are plentiful in Nature and in artificial systems. Illustrations can be encountered that range from physical and biological to technological and social sciences [1. Among the latter, one may speak of empirical data from scientific collaboration networks [2], cites of physics journals [3, the Internet traffic [4], Linux packages links [5], popularity of chess openings [6], as well as electoral results [7, 8], urban agglomerations [9, 10] and firm sizes all over the world [11. These systems lack a typical size, length or frequency for observables under scrutiny, a fact that usually leads to power law distributions

$$
p_{X}(x) \sim 1 / x^{1+\lambda},
$$

with $\lambda \geq 0$. The class of universality defined by $\lambda=1$, corresponding to the so-called Zipf's law (ZL) in the cumulative distribution or the rank-size distribution received much attention [3,6, 9, 15. Maillart et al. [5] have found that links' distributions follow ZL as a consequence of stochastic proportional growth. Such kind of growth assumes that an element of the system becomes enlarged proportionally to its size $k$, being governed by a Wiener process. The $\lambda=1$-class emerges from a condition of stationarity (dynamic equilibrium) [15. ZL also applies for processes involving either self-similarity [6] or fractal hierarchy [13, all of them mere examples amongst very general stochastic ones [14. The instance $\lambda=0$ is representative of a second universality-class following Costa Filho et al. 7, who studied vote-distributions in Brazil's electoral results. Therefrom emerge multiplicative processes in complex networks 8. $\lambda=0$-behavior ensues as well in i) city-population rank distributions [16, ii) Spanish electoral results [16, and iii) the degree distribution of social networks [17. As shown in Ref. [18, this universality class encompasses Benford's Law [19. In the present vein, still another kind of idiosyncratic distribution is often reported: the log-normal one [20, that has been observed in biology (length and sizes of living tissue 21]), finance (in particular the Black Scholes model [22]), and firms-sizes. The latter instance obeys Gibrat's rule of proportionate growth 24, that also applies to cities' sizes. Remarkably enough, all these variegated and sometimes quite complex systems share a scale-free growth behavior.

Together with geometric Brownian motion, there is a variety of models arising in different fields that yield Zipf's law and other power laws on a case-by-case basis [9, 10, 15, 25, 27, as preferential attachment [4] and competitive cluster growth 8 in complex networks, used to explain many of the scale-free properties of social, technological and biological networks. For instance we may mention urban dynamics [28, opinion dynamics [29, and electoral results [7, 30, that develop detailed realistic approaches. Ref. 31 is highly recommendable as a primer on urban modelling. Of course, the renormalization group is intimately related to scale invariance and associated techniques have been fruitfully exploited in these matters (as a small sample see [27, 32]).

Can the celebrated maximum entropy principle of Jaynes' (MaxEnt) 33, 34 explain the disparate phenomena recounted above? At first sight, one faces a seemingly unsurmountable difficulty. MaxEnt's variational principle, in conjunction with Shannon's logarithmic information measure, can not in straightforward fashion yield power laws (nor more involved combinations that include power laws), but only exponential distributions 34. This fact constituted one major motivation 35 for introducing Tsallis' information measure 36 (and its associated Tsallis-MaxEnt treatment 37]). One immediately gets power laws thereby as a result of varying the measure. We have shown in Ref. 38 how to overcome the above Shannonian limitation via suitable incorporation, into the MaxEnt principle, of dynamical information. As a consequence, we are now able to formulate a Shannonian Maximum Entropy approach [33, 34] common to all these systems. As stated in [38, this technique exhibits the peculiarity of including "equations of motion" as a part of the required a priori knowledge which always needs to be 
incorporated into the accompanying Jaynes' variational problem. The desired goal can in this way be successfully achieved, which provides a unifying "thermodynamiclike" explanation for the above mentioned disparate phenomena. The approach explicitly reconciles two apparent different viewpoints, those attached to i) growth models and ii) information-treatments 39.

\section{THEORETICAL PROCEDURE}

\section{General approach}

Accordingly, consider a rather general stochastic dynamical equation for the observable $x$, namely,

$$
\dot{x}(t)=k g[x(t)] ; g \text { arbitrary }
$$

where $k$ represents the derivative of a Wiener process (characterized by stationary independent increments) that frequently occurs in economic and social systems. We assume that there exists an appropriate transformation of $x$ that makes (2) invariant and a hallmark of the symmetry characterizing the system. Thermodynamics and many areas of physics have been shown by Frieden and Soffer 40] to be typified by translational symmetry if they are theoretically described à la Fisher [40]. This entails $g(x)=1$, which makes Eq. (2) the emblematic equation for linear Brownian motion. Our considerations will revolve around a new variable $u=u(x)$ such that $d x / d u=g(x)$, a variable that

1. should linearize the dynamic equation,

2. make the original $x$-symmetry a translational one $\dot{u}(t)=k$, and

3. according to the "central hypothesis" of Ref. 38, constitutes the tool for introducing dynamical information into MaxEnt by working with the $u$-entropy of the system.

One has 38

$$
S\left[p_{U}(u)\right]=\int_{\Omega} d u p_{U}(u) \log \left[p_{U}(u)\right],
$$

with $\Omega$ an appropriate "volume" in $u$-space. The equilibrium probability density $p_{U}(u)$ is derived from Jaynes' MaxEnt principle 33.

$$
\delta_{p}\left\{S\left[p_{U}(u)\right]-\sum_{i} \lambda_{i}\left\langle f_{i}(u)\right\rangle\right\}=0,
$$

where the mean values of the functions $f_{i}(u)$ describe the a-priori known constraints on the system, while $\lambda_{i}$ are the associated Lagrange multipliers. Our constraints represent conservation rules, operating on our system, that strongly condition the configuration of the equilibrium state. The general solution of the Jaynes problem is 33

$$
p_{U}(u) d u=\exp \left[-\sum_{i} \lambda_{i} f_{i}(u)\right] d u
$$

which, changing back to $x$ lead us to a $p_{X}(x)$ of the form

$$
p_{X}(x) d x=\exp \left[-\sum_{i} \lambda_{i} f_{i}(u(x))\right] \frac{d x}{g(x)} .
$$

The main difference with the usual MaxEnt (ME) solution is the Jacobian $d u / d x=1 / g(x)$. This looks trivial enough, but the Jacobian contains dynamical information, otherwise absent from the treatment. In a manner of speaking, we are thereby "extending" the exponentiallike form of the Jaynes' ME-solutions to other analytical possibilities.

\section{Scale-invariant systems}

To illustrate this procedure we consider the emblematic equation used in many models of mathematical finance (e.g., the Black-Scholes model [22]) and cities' and firms' sizes (Gibrat's law 23]), and also many of the "social" examples listed above. We speak then of the so-called geometrical Brownian motion:

$$
\dot{x}(t)=k x(t),
$$

which is symmetric under scale transformations $x^{\prime}=c x$ with $c$ an arbitrary constant. We are here defining a proportional growth or multiplicative process. A systems following such dynamics can be described by our approach. We set $u=\log \left(x / x_{0}\right)$, where $x_{0}$ is the minimum allowed value for $x$ (or a "reference"-one). The Jacobian of the transformation is $d u / d x=1 / x$, and the volume in $u$ space is defined as $\Omega$ : $\left[0 \leq u \leq u_{M}\right]$. Here $u_{M}=\log \left(x_{M} / x_{0}\right)$ corresponds to some maximum allowable value (which can be infinity). When no constraints are included and an infinite volume $\Omega$ is considered, the constituents of system diffuse as random walkers in $u$. Accordingly, the density distribution (DD) $p_{U}(u) d u$ is a gaussian distribution that becomes a log-normal one in $x$. This no-constraint case has been widely studied, and most city-population distributions follow it [23].

Let us discuss now just how adding constraints affect the equilibrium DD by considering the simple form $f_{i}(u)=u^{i}$. The case $i=0$ corresponds to normalization of the DD, while $i=1$ refers to having $\langle u\rangle$ as a constraint. We tackle the variational solution subjected to these two constraints, setting $\mu=\lambda_{0}$ and $\lambda=\lambda_{1}$. Accordingly, the equilibrium DD $p_{U}(u)$ extremizes the functional $F=S-\mu-\lambda\langle u\rangle$, so that

$$
p_{U}(u) d u=e^{-\mu-\lambda u} d u .
$$


The values for $\mu$ and $\lambda$ are obtained from the conservation rules $1=\int_{\Omega} d u e^{-\mu-\lambda u}$ and $\langle u\rangle=\int_{\Omega} d u e^{-\mu-\lambda u} u$ which yield $\langle u\rangle=e^{\mu}=1 / \lambda$ if $u_{M} \rightarrow \infty$. The $\mathrm{DD}$, as a function of $x$, becomes

$$
p_{X}(x) d x=e^{-\mu} x_{0}^{\lambda} \frac{d x}{x^{\lambda+1}},
$$

i.e., a power law whose exponent is characterized by $\lambda$, as that presented in Eq. (1). Look first at the particular solution $\lambda=0$ (no constraint on $\langle u\rangle$ ). One is led to $e^{\mu}=u_{M}$ and $\langle u\rangle=u_{M} / 2$. In terms of $x$ the DD is

$$
p_{X}(x) d x=\frac{1}{u_{M}} \frac{d x}{x} .
$$

As remarked above, such DD is related to Benford's Law. Using a thermodynamic analogy, the law describes the simple scenario of a non-interacting system confined to a finite volume of $u$-space, with a Gaussian distribution for $\dot{u}$, i.e., a scale-free ideal gas (SFIG) [16]. Even if the normalization seems to diverge here for $u_{M} \rightarrow \infty$, it can be kept finite in going to the thermodynamic limit (see [16): if $N$ is the total number of system's "elements", its density $\rho(x)=N p_{X}(x)$ is normalized in the limit $\left(N, u_{M} \rightarrow \infty\right)$ if $N / u_{M} \rightarrow \rho_{0}$, where $\rho_{0}$ is a constant. Let us pass now to the particular solution $\mu=0$ (relaxing the normalization constraint). If $u_{M} \rightarrow \infty$, we obtain from the conservation rules $\lambda=1$ and $\langle u\rangle=1$. Accordingly, the DD for the observable $x$ is

$$
p_{X}(x) d x=x_{0} \frac{d x}{x^{2}}
$$

i.e., Zipf's law (ZL). In a thermal context, the absence of normalization can be understood as an inability of the system to reach the thermodynamic limit. One may then speak of a Zipf regime [16]. The lack of normalization constraint is discussed also in Refs. [3, 14]. An explanation may be concocted: these elements might be distributed on the surface of an appropriate volume [3, 16. Accordingly, ZL usually holds for the upper tail and not for the "bulk" of the associated distribution. There is a free interchange of elements between the two regions with no "energetic cost" since the "chemical potential" $\mu$ is zero. Accordingly, the number of elements does not remain constant (photon statistics). This numberfluctuation has no effect in the bulk region where the thermodynamic limit is reached, but it totally determines how the density behaves on our putative "surface".

\section{PRESENT RESULTS: NUMERICAL EXPERIMENTS AND EMPIRICAL OBSERVATIONS}

So as to confirm the preceding MaxEnt predictions we have performed numerical experiments with random walkers and compared them with empirical citypopulation data. We solve numerically the equation
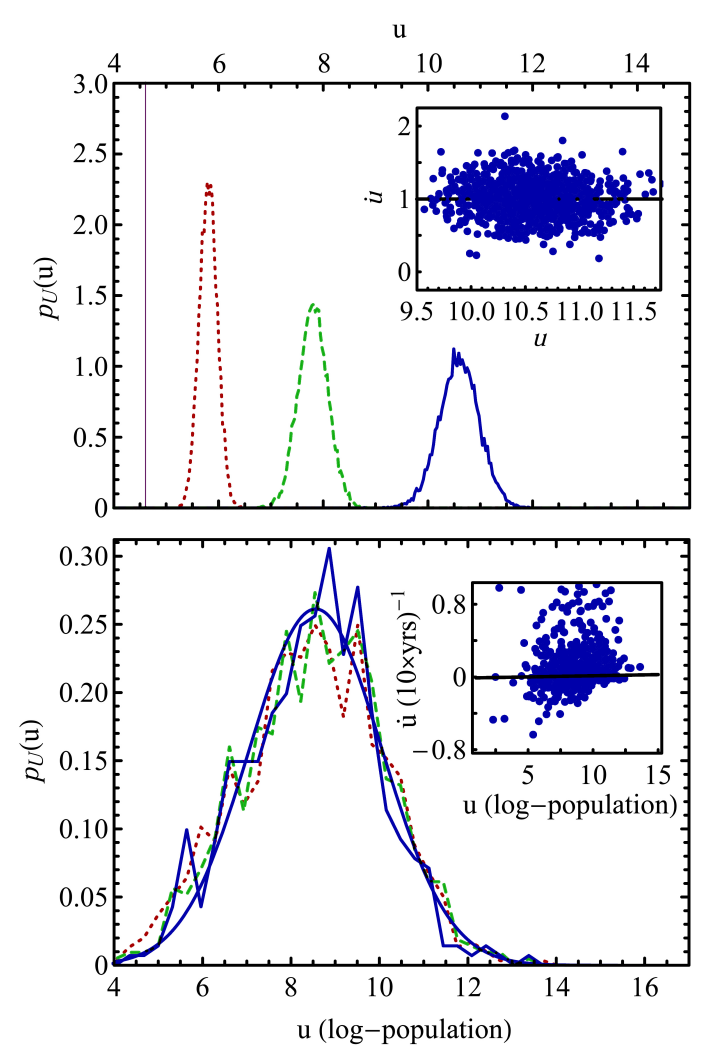

FIG. 1: Top panel: Evolution of a random walkers' distribution without constraints from an initial delta-one (purple solid line), passing through intermediate stages (red dotted, green dashed, and solid blue lines). Inset: logarithmic size $u$ vs. relative growth $\dot{u}$ (linear fit in black). Bottom panel: Florida's cities-population-distribution for 1990, 2000, and 2010 (same color-code as TP) compared with a normal distribution (smooth solid blue line). Inset: same as in top panel's inset.

$\dot{x}(t)=k x(t)$, discretizing the time in intervals of $\Delta t . k$ is randomly generated at each iteration from a Gaussian distribution with zero mean and variance $K$.

\section{Free evolution}

We first study evolution without constraints (as a control case), starting with $N=10000$ walkers located at the same position $x=100(u=2 \log 10)$, that evolve with $K=10$ and a time interval $\Delta t=10^{-5}$ in $x$. We also include a drift, as in Ref. [24. As expected, we obtain the diffusion process depicted in upper panel of Fig. 1 , which is a log-normal DD in $x$ (and a Gaussian in $u$ ). We now compare such evolution with that of the citiespopulation of Florida State using Census Bureau's data for the years 1990, 2000, and 2010 [41], also finding a $\log$-normal in $x$ with growing width $(1.64,1.65$ and 1.72 , respectively). The proportional growth condition is checked by computing the correlation between the log- 
arithm of the population of each place $u$ with its relative change $\dot{u}$, using two-points formulas for the time derivative. For $\dot{u}=k$ both observables are independent for scale-free systems. The correlation value is 0.027 , small enough to confirm that geometrical motion is taking place (see inset of Fig. 1, bottom panel). Even if the populations of Florida-cities do not seem to match the non-interacting assumption of our simulation, we expect a short-range nature for the correlation between the population of each pair of cities 42], relative to the State's size. According to our thermodynamic interpretation, we can think of a dilute scale-free gas at zero pressure and expanding freely.

\section{Evolution constrained by normalization and finite volume}

For an example with normalization constraint and finite volume $(\lambda=0)$, we arbitrarily define $x_{0}=1$, $x_{M}=10^{4},\left(u_{M}=4 \log 10\right)$ and use the same initial conditions as in the precedent case. Now, a "move" is not accepted if the new position falls outside the appropriate region. After some iterations the system reaches equilibrium, as shown in Fig. 2, top panel. We find a nice fitting of this equilibrium distribution to that predicted by MaxEnt [Eq. 10]], confirming the validity of our approach.

A system in this thermodynamical condition

1. obeys the proportional growth dynamics,

2. exhibits low correlation between its elements,

3. conserves the particle-number, and

4. is characterized by an objective, measurable sizeconstraint.

This last condition, for city-populations, has the form of a geographical constraint. Such is the case of, e.g., the Marshall Islands: this particular geographical area covers $181.3 \mathrm{~km}^{2}$ distributed into 29 atolls and 5 islands. Traversing the sea may reduce correlations between cities as compared to terra firma. The migration pattern concentrates in the two main population-centers, Rita and Ebeye, so that only indirect correlations are expected between the rest of the cities. We verified this issue by recourse to data from 1980, 1988, and 1999 [43. The islands' logarithmic population $u$, exhibits a correlation coefficient of $9 \times 10^{-5}$ with a relative increment $\dot{u}$, confirming the dynamics' nature (inset of Fig. 1b, bottom panel). We fitted the raw data for all low-correlated centers (154 populations) to the MaxEnt prediction and also to a log-normal with the same log-mean and log-variance that characterize the data (Fig. 1b, bottom panel). We found a correlation of 0.991 in the former case and of
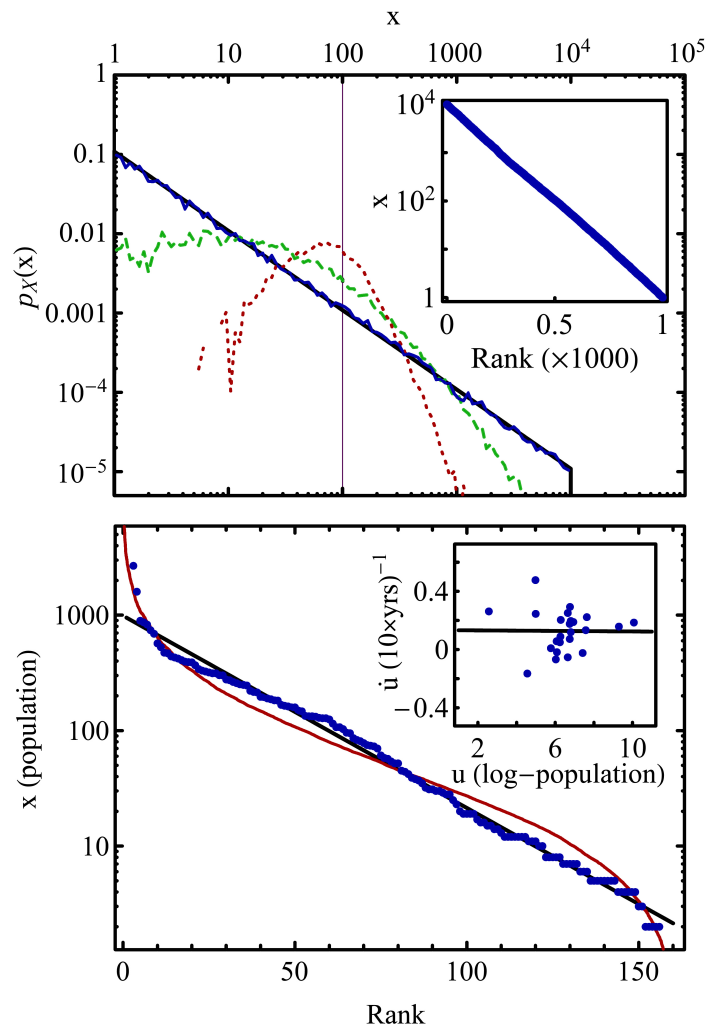

FIG. 2: Top panel: walkers' distribution for the first textexample $\lambda=0$ (origin of Benford Law). The blue solid line indicates convergence towards the MaxEnt-prediction (black solid line). Inset: equilibrium rank-distribution. Bottom Panel: rank-distribution of Marshall Islands' city-population versus i) MaxEnt-prediction for $\lambda=0$ (black line) and ii) a log-normal (red line). Inset: logarithmic size $u$ vs. relative growth $\dot{u}$ (linear fit in black).

0.979 in the latter one. One thus may visualize the Marshall Islands as a closed-volume scale-free ideal ga (SFIG) in thermal equilibrium, again empirically confirming the validity of our approach.

\section{Evolution constrained by a mean value-condition}

The second example presented here $(\mu=0)$ uses a simple algorithm that reproduces Zipf's Law. $N$ walkers "unfold" while guaranteeing the conservation laws' proper working: $\left\langle\log \left(x / x_{0}\right)\right\rangle=\langle u\rangle=1\left(\right.$ or $\sum_{i=1}^{N} \log \left(x_{i} / x_{0}\right)=$ $\left.\sum_{i=1}^{N} u_{i}=N\right)$. We start all the walkers at $x=e \times x_{0}$ (any distribution with $\langle u\rangle=1$ is adequate) and proceed iterating as follows:

1. select an arbitrary walker and change its position in proportional fashion according to $x(t+\Delta t)=$ $x(t) \times(1+k \Delta t)$, with a random value $k$;

2. assume that we have a bulk reservoir so that the exchange of elements has no 'energetic cost'. We 
randomly select a second walker and remove him from his position $x^{\prime}$;

3. add a new walker at a position that preserves the operating conservation rule, that is $x^{\prime}(t+\Delta t)=$ $x^{\prime}(t) \times(1-k \Delta t)$.

We iterate these steps till convergence is achieved. After some iterations, we do converge to the predicted MaxEnt distribution Eq. (11) (Fig. 3, top panel, with the same values for $x_{0}, N, K$, and $\Delta t$ used in the first example). thusly Once more, we reconfirm that the approach presented here works in reasonable fashion. Remarkably enough, a similar algorithm is also able to reproduce any arbitrary power law with exponent $\lambda+1$ by changing the value of the conservation rule in the fashion $\langle u\rangle=1 / \lambda$.

For city-populations, elements-exchange occurs in a continuous fashion. However the data are recorded at intervals of years, so that the ensuing exchange-effects are similar to those of our simulation. Areas belonging to the most-populated sites eventually change with time. Thus, we deal with an open system with no fixed number of elements, like our photon-gas above. As a well known example consider the most populated metropolitan USAareas [15]. We have confirmed the proportional growth hypothesis using data from years 1990, 2000, and 2010 [41] to find a small correlation of 0.016 between $u$ and $\dot{u}$ (inset of Fig. 1c, bottom panel). Our first $N=150$ areas are in Zipf's regime (Fig. 3, bottom panel) and constitute the surface of the statistical system at hand. We have verified the prevailing conservation rule, finding $\sum_{i=1}^{150} \log \left(x_{i} / x_{150}\right)=145.7,150.8$, and 154.9 for each year, respectively, close to the MaxEnt prediction of 150 . We have also verified that 10 of these areas (a 6.7\%) that in 1990 pertained to the Zipf regime are not characterized by it in 2010 .

\section{CONCLUSIONS}

Different phenomena involving scale-invariance can be unified via Jaynes' MaxEnt principle, provided that adequate dynamical information is suitably incorporated into the variational process, in the manner here prescribed. This allows one to perform a thermodynamic-like description of social systems, related to that of an ideal gas.

ACKNOWLEDGMENT: This work was partially supported by ANR DYNHELIUM (ANR-08-BLAN0146-01) Toulouse, project PIP1177 of CONICET, (Argentina), project FIS2008-00781/FIS (MICINN), and project FEDER (EU) (Spain, EU).
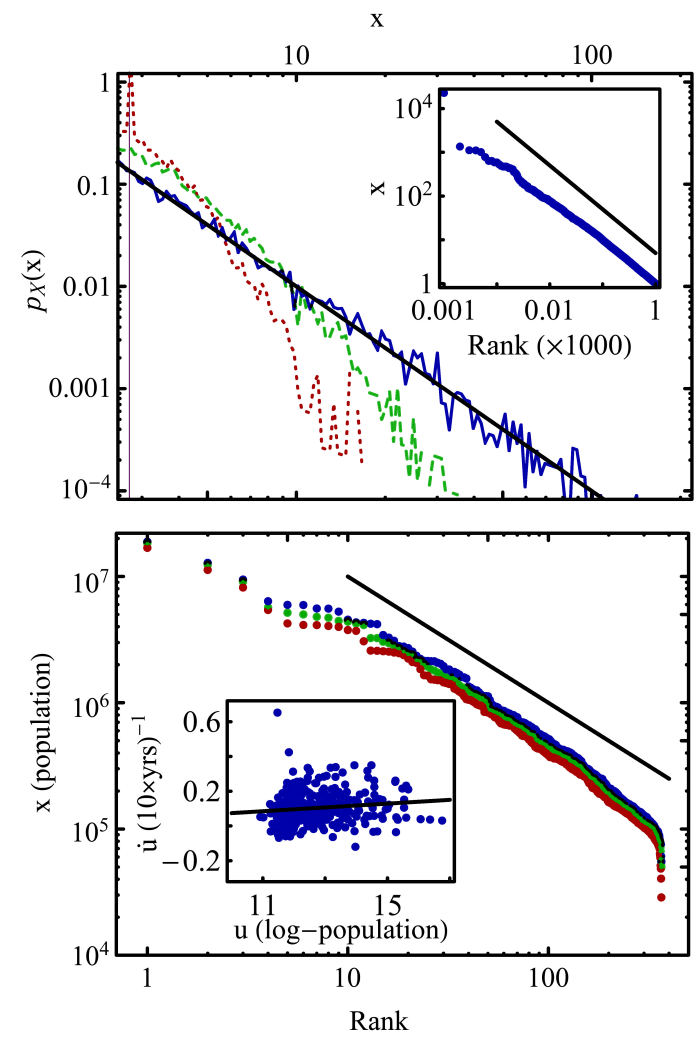

FIG. 3: Top panel: walkers distribution for the second textexample $\mu=0$ (Zipf's Law). Inset: equilibrium rankdistribution (slope $=1$ in black). Bottom panel: rankdistribution of USA's most-populated metropolitan areas for 1990, 2000, and 2010; Inset: logarithmic size $u$ vs. relative growth $\dot{u}$ (linear fit in black).

[1] M. Schroeder, Fractals, chaos and power laws (Freeman, NY, 1990).

[2] M. E. J. Newman, Phys. Rev. E 64, (2001) 016131.

[3] A. Hernando et al., A. Plastino, Phys. Lett. A 374, 18 (2009).

[4] A.-L. Barabasi, R. Albert, Rev. Mod. Phys. 74, 47 (2002).

[5] T. Maillart, et al., Phys. Rev. Lett. 101, 218701 (2008).

[6] B. Blasius, R. Tönjes, Phys. Rev. Lett. 103, 218701 (2009).

[7] R. N. Costa Filho, et al., Phys. Rev. E 60, 1067 (1999).

[8] A. A. Moreira, et al., Phys. Rev. E, 73, 065101(R) (2006).

[9] L. C. Malacarne, R. S. Mendes, and E. K. Lenzi, Phys. Rev. E 65, 017106 (2001).

[10] M. Marsili, Y. C. Zhang, Phys. Rev. Lett. 80, 2741 (1998).

[11] R. L. Axtell, Science 293, 1818 (2001).

[12] G. K. Zipf, Human Behavior and the Principle of Least Effort (Addison-Wesley, Cambridge, Mass., 1949).

[13] Y. Chen, Chaos Solitons Fract. 45, 63 (2012).

[14] B. C. Murtra, R.V. Solé, Phys. Rev. E 82, 011102 (2010).

[15] X. Gabaix, Quart. J. Econom. 114, 739 (1999). 
[16] A. Hernando, C. Vesperinas, A. Plastino, Physica A 389, 490 (2010).

[17] A. Hernando et al., Eur. Phys. J. B 76, 87 (2010).

[18] Weisstein, Eric W., Benford's Law from MathWorld.

[19] F. Benford, Proceed. Am. Phil. Soc. 78, 551572 (1938).

[20] Wikipedia http://en.wikipedia.org/wiki/Log-normal.

[21] J. S. Huxley, (1932) Problems of relative growth (Methuen \& Co. Lmtd., London, 1932).

[22] S. M. Ross, Introduction to Probability Models, 9th edition (Academis Press, NY, 2007).

[23] J. Eeckhout, Ame. Eco. Rev. 94, 1429 (2004).

[24] H. Rozenfeld, et al., Proc. Nat. Acad. Sci. 105, 18702 (2008).

[25] S. Ree, Phys. Rev. E 73 (2006) 026115.

[26] W. J. Reed, B. D. Hughes, Phys. Rev. E 66 (2002) 067103.

[27] S. Galam, J. Stat. Phys. 61, 943 (1990).

[28] UrbanSim: http://www.urbansim.org.

[29] C. Castellano, S. Fortunato, V. Loreto, Rev. Mod. Phys., 81, 591 (2009).

[30] S. Fortunato, C. Castellano, Phys. Rev. Lett. 99, 138701 (2007).
[31] M. Batty, Cities and Complexity (MIT Press, Cambridge, MA, 2005).

[32] S. Galam, Physica A 285, 66 (2000).

[33] A. Katz, Principles of stat. mechanics (Freeman, San Francisco, 1967).

[34] A. Katz, Principles of statistical mechanics (Freeman, San Francisco, 1967).

[35] A. Plastino, Physica A 344, 608 (2004).

[36] C. Tsallis, Introduction to Nonextensive Statistical Mechanics (Springer, NY, 2009).

[37] A. R. Plastino, A. Plastino, Phys. Lett. A 177, 177 (1993).

[38] A. Hernando, A. Plastino, A.R. Plastino, arXiv:1201.0889 and Eur. Phys. J. B (2012) in Press.

[39] H. Simon, Biometrika 42, 425 (1955).

[40] B.R. Frieden, B.H. Soffer, Phys. Rev. E 52, 2274 (1995).

[41] Census bureau, Government of USA (web).

[42] G. Krings et al., J. Stat. Mech, L07003 (2009).

[43] Econom. Pol. Plann. \& Stat. Office, Rep. of the Marshall Islands (web). 